\title{
Etude des réactions colorées de diverses souches de Candida
}

\author{
par M. QUILICI \\ Laboratoires de Parasitologie du C.H.U. et de la Faculté de Médecine de Marseille \\ (Professeurs: J. SAutet et J. RANQue)
}

\begin{abstract}
Résumé
100 souches de champignons levuriformes, dont 81 souches de Candida, ont été testées sur trois milieux réactifs colorés. Tetrazolium, Nickerson-Cator, Arbutine. Les résultats obtenus sont rapportés sous forme de tableau.
\end{abstract}

\section{Summary}

We have tested a hundred strains of Yeasts, from which about eighty-one strains of Candida. We use three different reactive colored media: Tetrazolium, Nickerson-Cator, Arbutin. Now our results are summarized and presented on a table. 


\begin{tabular}{|c|c|c|c|}
\hline Groupe $C$. albicans & Tetrazolium & NICKERSON-CATOR & ARbUtine \\
\hline $\begin{array}{l}\text { C. albicans, } 35 \text { souches } \\
\text { (dont } 1 \text { C. aldoi) ..... }\end{array}$ & $\begin{array}{l}\text { blanc à blanc très } \\
\text { légèrement rosé }\end{array}$ & $\begin{array}{l}32 \text { souches noir } \\
3 \text { souches brun } \\
\text { plus ou moins clair }\end{array}$ & négative \\
\hline C. truncata, 1 souche . & blanc & noir & négative \\
\hline C. triadis, 2 souches .. & blanc & noir & négative \\
\hline $\begin{array}{l}\text { C. stellatoidea, } 3 \text { sou- } \\
\text { ches } \ldots \ldots \ldots \ldots \ldots \ldots\end{array}$ & blanc & noir & négative \\
\hline $\begin{array}{l}\text { C. tropicalis (dont } 3 \\
\text { chalmersi) souches } \ldots . .\end{array}$ & rose violet & noir chocolat & négative \\
\hline $\begin{array}{l}\text { C. pseudotropicalis, } \quad 5 \\
\text { souches } \ldots \ldots \ldots \ldots \ldots\end{array}$ & blanc rosé & brun à marron & positive \\
\hline C. krusei, 5 souches ... & blanc à rosé & $\begin{array}{l}3 \text { souches noires } \\
2 \text { souches beiges }\end{array}$ & négative \\
\hline $\begin{array}{l}\text { C. parapsilosis, } 6 \text { sou- } \\
\text { ches } \quad \ldots \ldots \ldots \ldots \ldots \ldots\end{array}$ & banc à rosé & beige à brun & négative \\
\hline $\begin{array}{l}\text { C. guillermondi, } 4 \text { sou- } \\
\text { ches } \ldots \ldots \ldots \ldots \ldots \ldots \ldots\end{array}$ & rose violet à violet & beige chamois & positive \\
\hline C. utilis, 1 souche $\ldots$. & blanc rosé & noir & négative \\
\hline $\begin{array}{l}\text { C. mesenterica (C. pa- } \\
\text { rakrusei ?), } 1 \text { souche } .\end{array}$ & violet & brun clair & positive \\
\hline C. brumpti, 2 souches. & blanc rosé & noir & négative \\
\hline $\begin{array}{l}\text { C. zeylanoides, } 4 \text { sou- } \\
\text { ches } \ldots \ldots \ldots \ldots \ldots \ldots\end{array}$ & rosé à corail & brun & $\begin{array}{l}\text { légèrement } \\
\text { positive }\end{array}$ \\
\hline C. lipolytica, 1 souche . & rose & brun & négative \\
\hline $\begin{array}{l}\text { C. macedoniensis, } 2 \text { sou- } \\
\text { ches } \ldots \ldots \ldots \ldots \ldots \ldots\end{array}$ & rose violet & brun foncé & positive \\
\hline C. curvata, 3 souches. & violet & marron noir & positive \\
\hline C. rugosa, 2 souches. & rose corail & noir & négative \\
\hline C. mycoderma, 1 souche & rosé & beige clair & négative \\
\hline
\end{tabular}


Souches autres que les Candida

\begin{tabular}{|c|c|c|c|}
\hline & TÉTRAZOLIUM & NICKERSON-CATOR & ARBUtine \\
\hline $\begin{array}{l}\text { D. kloeckeri (C. flareri), } \\
1 \text { souche } \ldots \ldots \ldots \ldots \ldots\end{array}$ & rose & brun clair & $\begin{array}{l}\text { légèrement } \\
\text { positive }\end{array}$ \\
\hline C. neoformans, 1 souche & blanc rosé & crème clair & $\begin{array}{c}\text { positive } \\
\text { tardivement }\end{array}$ \\
\hline S. cerevisiae, 3 souches & blanc rosé & négatif & négative \\
\hline $\begin{array}{l}\text { Geotrichum } S p ., 3 \text { sou- } \\
\text { ches } \ldots \ldots \ldots \ldots \ldots \ldots\end{array}$ & rose & beige crème & négative \\
\hline $\begin{array}{l}\text { Trichosporon } S p ., 1 \text { sou- } \\
\text { che } \quad \ldots \ldots \ldots \ldots \ldots \ldots \ldots\end{array}$ & rose & marron & négative \\
\hline $\begin{array}{l}\text { Torulopsis molischiana, } \\
1 \text { souche } \ldots \ldots \ldots \ldots \ldots\end{array}$ & rose pâle & crème clair & positive \\
\hline $\begin{array}{l}\text { Torulopsis magnoliae, } \\
1 \text { souche } \ldots \ldots \ldots \ldots \ldots\end{array}$ & blanc & négatif & négative \\
\hline $\begin{array}{l}\text { Torulopsis } S p ., \quad 2 \text { sou- } \\
\text { ches } \ldots \ldots \ldots \ldots \ldots \ldots\end{array}$ & blanc rosé & brun clair & négative \\
\hline
\end{tabular}

L'étude de la réduction des sels de Tetrazolium et l'utilisation du milieu de Nickerson-Cator sont d'introduction relativement récentes dans le diagnostic des Candida.

Nous avons pensé qu'il serait intéressant de tester vis-à-vis de ces deux milieux l'ensemble des souches de notre collection de levures.

Nous y avons ajouté un test coloré beaucoup plus ancien, le milieu à l'Arbutine. 100 souches diverses ont été ainsi ensemencées sur ces trois milieux.

\section{Technique utilisée.}

Ces divers milieux étaient coulés en boîte de Pétri. Une goutte d'une suspension légère de chacune des levures était déposée sur ce milieu. On réalisait ainsi cinq ensemencements par boîte.

Les lectures étaient effectuées à $24-48$ et 72 heures pour les milieux au Tétrazolium et de Nickerson-Cator. Le troisième jour pour le milieu à l'Arbutine.

\section{Résultats.}

Les résultats sont consignés dans le tableau ci-joint. 


\section{Conclusions.}

Une étude du genre Candida est actuellement en cours, dans notre laboratoire, nous ne tirerons donc aucune conclusion quant aux synonymies de certaines de nos souches.

Les milieux à l'Arbutine et au Tétrazolium sont maintenant bien connus et nous nous contenterons de rapporter les résultats obtenus sans autres commentaires.

A propos du milieu de Nickerson-Cator nous pensons pouvoir affirmer que ce milieu n'est absolument pas spécifique de Candida albicans. Il ne semble devoir être considéré que comme une simple réaction colorée complémentaire au même titre que le Tétrazolium ou l'Arbutine. A ce sujet, nous avions, parallèlement aux ensemencements sur boîte de Pétri, étudié les mêmes souches sur les petits tubes de ce même milieu trouvés dans le commerce sous le nom de Candalbi-test. L'ensemencement y est beaucoup plus délicat et la lecture très malaisée.

Il semble préférable de couler le milieu de Nickerson-Cator en tube de 18 ou en boîte de Pétri. 